\title{
Cierre de comunicación interventricular en menores de un año en un hospital público
}

\author{
Ventricular septal defect repair in children during first year of \\ life in a public hospital
}

\author{
Dra. Verónica Becerra ${ }^{a}$ Dra. María Althabe ${ }^{a}$, Dra. Gladys Salgado ${ }^{b}$, Dr. Jorge Barrettac, \\ Dr. Javier Cornelis ${ }^{c}$, Dr. Pablo García Delucis ${ }^{c}$ y Dr. Ricardo Magliola ${ }^{a}$
}

\section{RESUMEN}

Introducción. La comunicación interventricular (CIV) es la cardiopatía congénita más frecuente y el cierre quirúrgico primario es la estrategia de elección para corregirla. El objetivo es describir los resultados de la reparación quirúrgica en menores de 1 año y analizar factores de riesgo de morbilidad y mortalidad.

Pacientes y métodos. Estudio retrospectivo; se incluyeron todos los pacientes con CIV operados entre 2004 y 2011. Se registraron variables demográficas, de la cirugía y del posoperatorio: edad, peso, síndrome genético, tipo de CIV, días de internación, complicaciones y evolución. Como factores de riesgo de mortalidad y morbilidad, se analizaron edad $<6 \mathrm{~m}$, peso $<3$ $\mathrm{kg}$, síndrome de Down, desnutrición e infección respiratoria previa.

Resultados. Se operaron 256 pacientes, con edad de 5,3 meses ( $21 \mathrm{~d}-1 \mathrm{a}$ ), peso de $4,75 \mathrm{~kg}$ (2,2-13), 32\% con síndrome de Down y 17,5\% con ventilación mecánica preoperatoria. La CIV tipo perimembranosa fue la más frecuente $(62 \%)$. El $28 \%$ presentó alguna complicación y el $7 \%$ requirió reoperación por sangrado, infección o defecto. La mediana de internación fue de 6 días (1-185). Se registró una mortalidad posoperatoria de $3 \%$. La desnutrición, edad $<6 \mathrm{~m}$, peso $<3 \mathrm{~kg}$ y la infección respiratoria previa se asociaron a una internación prolongada. No se identificaron factores de riesgo para la mortalidad.

Conclusión. En nuestra institución, el cierre quirúrgico primario de la CIV es un procedimiento con resultados satisfactorios.

a. Unidad de Cuidados Intensivos Pediátricos 35.

b. Servicio de Cardiología.

c. Servicio de Cirugía Cardiovascular.

Hospital de Pediatría

"Prof. Dr. Juan P.

Garrahan".

\section{Correspondencia:}

Dra. Verónica Becerra: draverobecerra@yahoo. com.ar

Conflicto de intereses: Ninguno que declarar.

Recibido: 8-4-2014 Aceptado: 25-8-2014 and respiratory infection prior syndrome were analyzed.

Results. 256 patients, age 5.3 months (21d$1 \mathrm{y})$, weight $4.75 \mathrm{~kg}$ (2.2 to 13$), 32 \%$ with Down syndrome and $17.5 \%$ with preoperative mechanical ventilation were operated. Perimembranous VSD was the most frequent type (62\%). $28 \%$ experienced complications and $7 \%$ required reoperation for bleeding, infection or defect. The median hospital stay was 6 days (1-185). Postoperative 30 days mortality was $3 \%$. Age $<6 \mathrm{~m}$, weight $<3 \mathrm{~kg}$, malnutrition and prior respiratory viral infection were associated with prolonged hospitalization, but no risk factors for mortality were identified.

Conclusion. The primary surgical closure of the VSD is a procedure with satisfactory results at our institution.

Key words: congenital heart disease, ventricular septal defect, primary surgical closure.

http:/ /dx.doi.org/10.5546/aap.2014.548

\section{INTRODUCCIÓN}

A partir de la reducción de las causas infecciosas, las malformaciones congénitas en general y las cardiopatías congénitas en particular han adquirido mayor relevancia como causa de mortalidad infantil. ${ }^{1} \mathrm{La}$ comunicación interventricular (CIV) es la cardiopatía congénita más frecuente y ocurre aproximadamente en 2:1000 recién nacidos vivos. Es la causa más frecuente de insuficiencia cardíaca en niños de más de dos semanas de vida. Si bien existe tratamiento médico que puede mejorar los síntomas, es indudable que el cierre quirúrgico primario en los primeros meses de vida es la estrategia de elección para reparar esta cardiopatía. El desarrollo y la mejora de los procedimientos perioperatorios han permitido que la mayoría de estos pacientes tengan resultados satisfactorios en cuanto 
a sobrevida y calidad de vida. ${ }^{2,3}$ Dado que los pediatras participan en el cuidado preoperatorio de estos pacientes, es importante que conozcan los resultados actuales de este tipo de cirugías, así como los factores de riesgo en nuestra población y la frecuencia de complicaciones.

El objetivo de este estudio es describir los resultados de la reparación quirúrgica de la CIV única en menores de 1 año en un centro público de alta complejidad y analizar los factores de riesgo de morbilidad y mortalidad en nuestra población.

\section{PACIENTES Y MÉTODOS}

Estudio descriptivo retrospectivo; se incluyeron todos los pacientes con diagnóstico de CIV única aislada o asociada a comunicación interauricular (CIA), foramen oval (FO) y/o ductus, sometidos a reparación quirúrgica entre enero de 2004 y diciembre de 2011. Se registraron variables demográficas: sexo, edad, peso, síndrome genético, tipo de CIV, presencia de cirugías cardíacas previas y condición preoperatoria; variables de la cirugía: tiempo de circulación extracorpórea (CEC) y tiempo de clampeo (TC); y del posoperatorio: días de internación, días de ventilación mecánica (VM), cierre esternal diferido, complicaciones y mortalidad posoperatoria temprana ( $<30$ días).

Se define condición preoperatoria desfavorable a la presencia de, al menos, dos condiciones: desnutrición grave, ventilación mecánica preoperatoria, drogas inotrópicas intravenosas y/o aislamiento de virus respiratorios dentro de los 30 días previos a la cirugía. Se considera desnutrición grave a más de 2 DE (desvíos estándar) por debajo del peso para la edad.

Se analizaron como potenciales factores de riesgo de mortalidad y morbilidad la edad $<6 \mathrm{~m}$, el peso $<3 \mathrm{~kg}$, la presencia de síndrome genético asociado y el aislamiento de virus respiratorios dentro de los 30 días previos a la cirugía. ${ }^{4-7}$

Se consideró complicación aquella condición que implicara tratamiento adicional y/o prolongación de internación y/o riesgo potencial de vida para el paciente.

Las complicaciones se diferenciaron en vinculables al procedimiento (CIV residual, arritmias, bloqueo auriculoventricular) y vinculables a la cirugía cardíaca en general (infecciones, trombosis, estenosis subglótica, parálisis diafragmática, etc.).

Los datos fueron obtenidos de las historias clínicas y de la base de datos del servicio.
El protocolo fue aprobado por el Comité de Investigación del hospital (n 810/2014); por tratarse de un estudio retrospectivo sin intervención, en el cual no se reportan datos que identifiquen a los pacientes, el Comité de Ética no consideró imprescindible contar con el consentimiento informado.

Se realizó un análisis estadístico descriptivo; los resultados se reportan como mediana y rango o media y DE para variables continuas y números absolutos y porcentaje para las discretas. Se realizó un análisis univariado de los factores de riesgo de mortalidad y de internación $>6$ días como variable subrogante de morbilidad, con la prueba de $\mathrm{Chi}^{2}$ o Rank sum test, según correspondiese (Stata10.0). Este punto de corte surge de agregar un día más a la mediana histórica de internación de la unidad. ${ }^{8}$

\section{RESULTADOS}

En el período de estudio, 256 pacientes fueron sometidos al tratamiento quirúrgico de cierre de CIV única. Los datos demográficos se describen en la Tabla 1. El tipo de CIV más frecuente fue perimembranosa en 159 pacientes (62\%); tipo muscular en 23 (9\%); tipo subpulmonar en 13 (5\%); tipo canal en $6(3 \%)$; y no especificada en 53 (21\%). En 158 pacientes, la CIV estaba asociada a ductus (145), CIA o foramen oval permeable (89), o ambas (146). Solo dos pacientes tenían cirugía previa (1 con ligadura de ductus y 1 con cerclaje de arteria pulmonar). El 17,5\% de los pacientes (45) presentaban VM preoperatoria, 9 con infección respiratoria asociada y 12 con desnutrición grave al momento de la cirugía.

En relación con el procedimiento quirúrgico, el tiempo de CEC promedio fue $67 \pm 21 \mathrm{~min}$

Tabla 1. Demografía

\begin{tabular}{lc}
\hline & $\mathbf{n}=\mathbf{2 5 6}$ \\
\hline Edad (mediana y rango) & $5,3 \mathrm{~m}(21$ días-1 año) \\
Peso (mediana y rango) & $4,75 \mathrm{~kg}(2,2-13)$ \\
Sexo femenino & $140(54 \%)$ \\
Síndrome de Down & $82(32 \%)$ \\
Pacientes con VM preoperatoria & $45(17,5 \%)$ \\
Tiempo de bomba (media y DE) & $67 \pm 21 \mathrm{~min}$ \\
Tiempo de clampeo (media y DE) & $47 \pm 21 \mathrm{~min}$ \\
Días de internación (mediana y rango) & 6 días (1-185) \\
\hline
\end{tabular}

VM: ventilación mecánica. 
y el de clampeo, de $47 \pm 21 \mathrm{~min}$. La vía de abordaje fue por auriculotomía derecha en la mayoría de los pacientes $(67,5 \%)$, seguido de la vía transpulmonar (3\%), vía combinada $(2,7 \%)$ y solo $0,4 \%$ por ventriculotomía. En el $68 \%$ de los casos, el cierre se realizó con parche de pericardio; en $6,5 \%$, cierre con puntos de sutura; en $0,8 \%$, con parche de goretex; en $0,4 \%$, con técnicas combinadas; $y$, en $24 \%$ de los casos, no se especificó. En cuatro pacientes (1,5\%), se difirió el cierre esternal como estrategia de recuperación posoperatoria. En los pacientes que presentaban CIA, foramen oval y/o ductus, se realizó su reparación en el mismo tiempo quirúrgico.

En cuanto a la evolución posoperatoria, 176 pacientes $(69 \%)$ requirieron VM con una mediana de dos días (1-62). La mediana de internación posoperatoria fue de 6 días (1-185), con una frecuencia de complicaciones de $28 \%$ de los casos, algunos de ellos con más de una. No se realizó discriminación entre el tiempo de internación en la Unidad de Cuidados Intensivos (UCI) y la sala dado que, por razones inherentes a la institución, la mayoría de nuestros pacientes egresan del hospital desde la UCI (73\%), aunque el requerimiento habitual de cuidados intensivos en esta patología no suele exceder las $48 \mathrm{~h}$. Se reoperaron por diferentes causas 18 pacientes (7\%) (Tablas 2 y 3). La mortalidad posoperatoria fue $3 \%$, cuatro pacientes fallecieron dentro de las

TABla 2. Complicaciones en orden de frecuencia

\begin{tabular}{lcc}
\hline Complicación & $\mathbf{n}$ & \% \\
\hline Bacteriemia/sepsis & 15 & 5,8 \\
Infección de herida quirúrgica & 11 & 4,3 \\
Arritmias & 11 & 4,3 \\
Neumotórax & 9 & 3,5 \\
Obstrucción alta posextubación & 8 & 3,1 \\
Sangrado posoperatorio & 5 & 1,9 \\
Convulsiones & 5 & 1,9 \\
Infección respiratoria & 4 & 1,5 \\
Quilotórax & 4 & 1,5 \\
Endocarditis & 2 & 0,8 \\
Trombosis & 2 & 0,8 \\
Parálisis frénica & 1 & 0,4 \\
Otras & 8 & 3,1 \\
Total & 85 & 33 \\
\hline
\end{tabular}

Dado que hay pacientes con más de una complicación, el $\mathrm{n} .^{\circ}$ de complicaciones supera al $n .^{\circ}$ de pacientes complicados (71). primeras $24 \mathrm{~h}$ por complicaciones quirúrgicas (3) e hipertensión pulmonar (1), y los otros 4 fallecieron en el posoperatorio alejado por complicaciones infecciosas asociadas a mala condición preoperatoria (VM y desnutrición grave). Los factores de riesgo analizados no se asociaron de manera estadísticamente significativa con mayor mortalidad, pero todos ellos, excepto el síndrome de Down, lo hicieron con internación prolongada (Tablas 4 y 5).

TABLA 3. Causas de reoperación

\begin{tabular}{lcc}
\hline Causa & $\mathbf{n}$ & $\mathbf{\% ( * )}$ \\
\hline CIV residual & 3 & 1,17 \\
Marcapasos definitivo & 2 & 0,78 \\
Sangrado posoperatorio & 1 & 0,39 \\
Plicatura diafragmática & 1 & 0,39 \\
Mediastinitis & 5 & 1,95 \\
Anillo vascular & 1 & 0,39 \\
Quilotórax & 1 & 0,39 \\
Derrame pericárdico & 1 & 0,39 \\
Otras & 3 & 1,17 \\
Total & 18 & 7 \\
\hline
\end{tabular}

(*) El \% se calcula sobre el total de pacientes operados (256).

CIV: comunicación interventricular.

TABLA 4. Factores de riesgo de mortalidad

\begin{tabular}{lccc}
\hline & $\mathbf{n}$ & Fallecidos & $\mathbf{p}$ \\
\hline Edad $<6 \mathrm{~m}$ & 147 & 4 & 0,66 \\
Peso $<3 \mathrm{~kg}$ & 24 & 0 & 0,35 \\
Síndrome de Down & 82 & 0 & 0,05 \\
IRAB previa & 9 & 0 & 0,58 \\
Desnutrición & 12 & 1 & 0,28 \\
\hline
\end{tabular}

IRAB: infección respiratoria aguda baja.

TABla 5. Factores de riesgo de internación mayor de 6 días

\begin{tabular}{lccc}
\hline & $\mathbf{n}$ & Internación $>\mathbf{6}$ días & $\mathbf{p}$ \\
\hline Edad $<6 \mathrm{~m}$ & 147 & 76 & 0,03 \\
Peso $<3 \mathrm{~kg}$ & 24 & 18 & 0,001 \\
Síndrome de Down & 82 & 41 & 0,18 \\
IRAB previa & 9 & 8 & 0,005 \\
Desnutrición & 12 & 2 & 0,005 \\
\hline
\end{tabular}

IRAB: infección respiratoria aguda baja. 


\section{DISCUSIÓN}

Hasta donde hemos podido identificar, este estudio constituye la serie más grande de reparación de CIV publicada en una sola institución en nuestro país. El Hospital de Pediatría "J. P. Garrahan" es un centro de alta complejidad, que realiza 700 procedimientos de cirugía cardiovascular por año, que recibe pacientes de todo el país y es un centro de capacitación y entrenamiento para profesionales del país y la región.

Por tratarse de una institución pública, que recibe pacientes de todo el país y en diversa condición clínica, creemos que los resultados pueden considerarse una muestra representativa de los que se pueden alcanzar en esta patología.

La mortalidad de la serie es comparable con series internacionales $y$, en los últimos 2 años, se ha reducido al $1 \% .^{3}$ La morbilidad es algo elevada, pero no se aleja de lo que reporta la bibliografía. El porcentaje de pacientes con bloqueo auriculoventricular (bloqueo AV) completo que requiere marcapaso definitivo, una de las complicaciones que podría desalentar el cierre primario, es menor del 1\%; las restantes complicaciones reportadas son comunes a cualquier tipo de cirugía cardíaca. La infección de herida quirúrgica fue la causa más frecuente de reoperación, así como las infecciones constituyeron más del 50\% de las complicaciones. Esto podría estar asociado a la mala condición preoperatoria de muchos pacientes, a las dificultades en el proceso de derivación de los pacientes y a una insuficiente oferta de turnos quirúrgicos que implican, a veces, internaciones preoperatorias prolongadas. El sangrado posoperatorio tampoco constituyó una complicación frecuente y menos del 1\% requirió cirugía por esta causa.

La proporción de pacientes con síndrome de Down fue más alta que en series similares publicadas, pero, al igual que en ellas, no significó un factor de riesgo de mortalidad ni de internación prolongada, aunque esto último sí se menciona en algunas de las series publicadas. ${ }^{9}$

Cabe destacar que, en esta serie, solo dos pacientes tuvieron un cerclaje previo a la reparación: un caso realizado en otra institución y otro con una CIV muy amplia, a la que se realizó un cerclaje y, por mala evolución, se decidió la reparación completa en la misma internación, con evolución favorable. En décadas pasadas, era habitual recurrir al cerclaje de la arteria pulmonar, sobre todo en lactantes pequeños, para reducir el flujo pulmonar y permitir el crecimiento hasta la reparación definitiva. Con la cirugía paliativa, no siempre se alcanza este objetivo, puede distorsionar las ramas pulmonares y obliga al paciente a enfrentar una nueva cirugía. En nuestra institución, esta estrategia solo se reserva para casos en los que el cierre es extremadamente dificultoso o técnicamente imposible y en pacientes con CIV múltiple. Con la reparación primaria del defecto, se logra restituir tempranamente la fisiología normal y así reducir el impacto de la malformación en el crecimiento y desarrollo del niño.

Otro aspecto importante para comentar es la evolución de los pacientes que presentaron infección respiratoria preoperatoria. Si bien la recomendación es alejar la cirugía con CEC del episodio agudo, estos pacientes se encontraban en VM sin posibilidades de extubación. ${ }^{7} \mathrm{La}$ estrategia en este caso fue esperar una disminución razonable de los requerimientos de VM para realizar la cirugía. Estos pacientes tuvieron internaciones más prolongadas, pero no mostraron mayor mortalidad. Cabe mencionar que el análisis de factores de riesgo fue solo univariado, por lo que no pueden descartarse posibles confundidores.

En función de estos resultados, creemos que no hay beneficio en diferir la reparación definitiva en pacientes sintomáticos.

\section{CONCLUSIÓN}

El cierre primario de la comunicación interventricular en pacientes menores de 1 año es un procedimiento con resultados satisfactorios. En nuestra serie, la edad $<6 \mathrm{~m}$, el peso $<3 \mathrm{~kg}$, la desnutrición y la infección respiratoria previa se asociaron de manera significativa con una internación prolongada, pero no con una mayor mortalidad. La presencia de síndrome de Down no representó un factor de riesgo para ninguna de las dos condiciones analizadas.

\section{REFERENCIAS}

1. Unicef, Sociedad Argentina de Pediatría. Salud maternoinfanto-juvenil en cifras 2013. Buenos Aires: SAP; 2013. Tabla 18: Diez principales causas de muertes posneonatales. República Argentina, 2011. [Disponible en: http:// www.unicef.org/argentina/spanish/salud_SapUnicef_ cifras2013.pdf]. [Consulta: 18 de febrero de 2014].

2. Scully BB, Morales DL, Zafar F, McKenzie ED, et al. Current expectations for surgical repair of isolated ventricular septal defects. Ann Thorac Surg 2010;89(2):544-9.

3. Anderson BR, Stevens KN, Nicolson SC, Gruber SB, et al. Contemporary outcomes of surgical ventricular septal defect closure. J Thorac Cardiovasc Surg 2013;145(3):641-7. 
4. Lacour-Gayet F, Clarke D, Jacobs J, Comas J, et al. The Aristotle score: a complexity-adjusted method to evaluate surgical results. Eur J Cardiothorac Surg 2004;25(6):911-24.

5. O’Brien SM, Clarke DR, Jacobs JP, Jacobs ML, et al. An empirically based tool for analyzing mortality associated with congenital heart surgery. J Thorac Cardiovasc Surg 2009;138(5):1139-53.

6. Jenkins KJ, Gauvreau K, Newburger JW, Spray TL, et al. Consensus-based method for risk adjustment for surgery for congenital heart disease. J Thorac Cardiovasc Surg 2002;123(1):110-8.
7. Newth C, Hammer J. Pulmonary Issues. En: Chang A Hanley F, Wernovsky G, Wessel DL, eds. Pediatric Cardiac Intensive Care. Baltimore: Lippincott Williams \& Wilkins; 1998:351-67.

8. Magliola RH, Althabe M, Moreno G, Lenz AM, et al. Cardiopatías congénitas: resultados quirúrgicos en un hospital público en Argentina. Arch Cardiol Mex 2011;81(3):178-82.

9. Fudge JC Jr, Li S, Jaggers J, O’Brien SM, et al. Congenital heart surgery outcomes in Down syndrome: analysis of a national clinical database. Pediatrics 2010;126(2):315-22.

\section{Archivos hace 75 años}

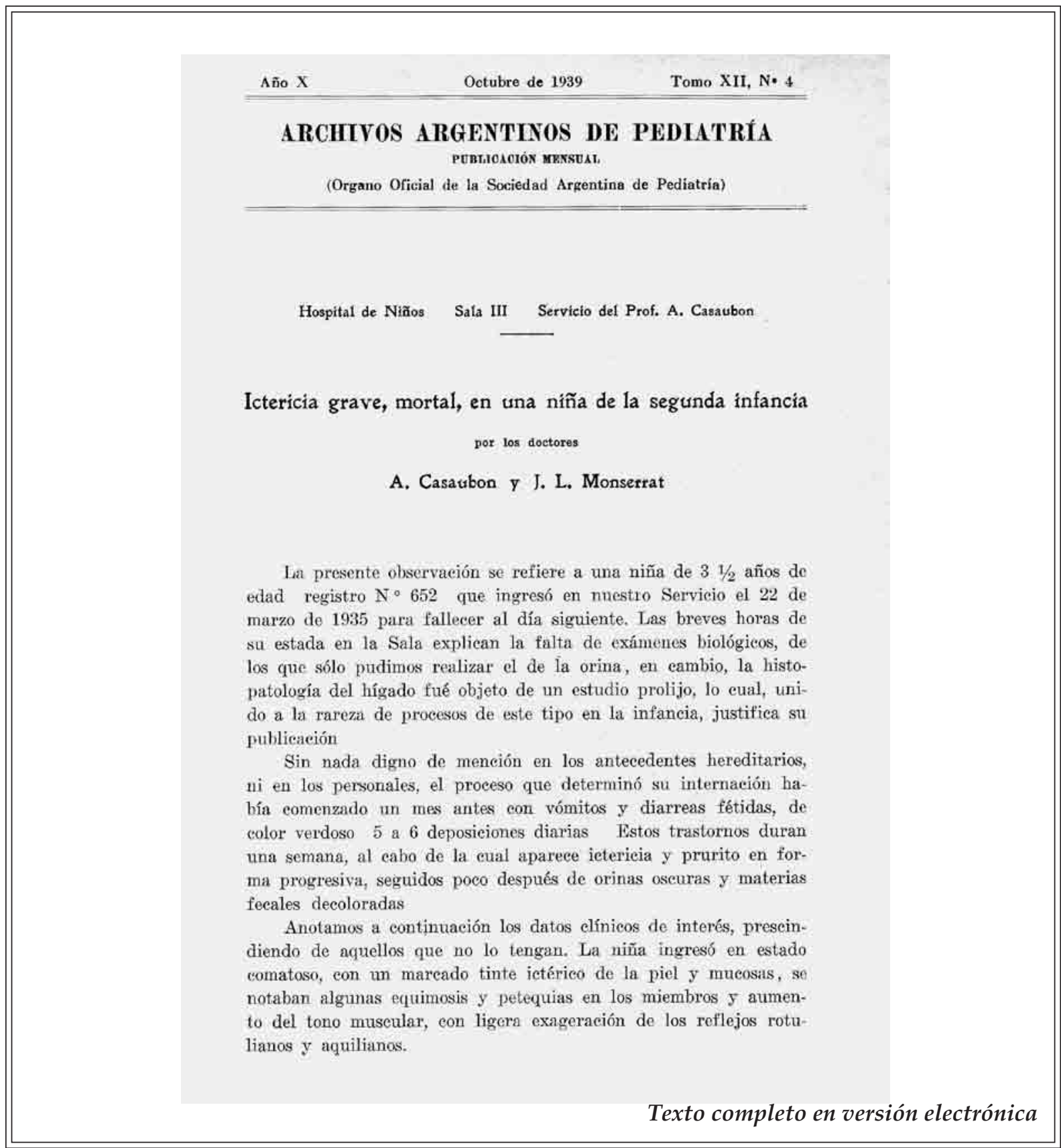

